\title{
Ökonomische Analyse dreier technischer Anlagen hinsichtlich deren Eignung für Predictive Maintenance
}

\author{
Eine multiple Fallstudie am Flughafen München
}

Julian Rott ${ }^{1} \cdot$ Sebastian Floerecke ${ }^{2}$ Christoph Ertl ${ }^{1}$ Alexander Herzfeldt ${ }^{1} \cdot$ Markus Böhm $^{1} \cdot$ Helmut Krcmar $^{1}$

Angenommen: 10. April 2021 / Online publiziert: 1. Juni 2021

(c) Der/die Autor(en) 2021

\section{Zusammenfassung}

Treten bei technischen Anlagen während ihres Lebenszyklus Fehler auf, ist dies in den meisten Fällen das Ergebnis eines schleichenden Prozesses und nicht eines plötzlich auftretenden Ereignisses. Mithilfe geeigneter Verfahren können Fehler bereits vor ihrem Eintritt erkannt werden. Die von Herstellern vorgegebene Instandhaltungsstrategie berücksichtigt diese Möglichkeit für gewöhnlich jedoch nicht. Im Unterschied dazu zielt Predictive Maintenance (PdM) auf die Durchführung von Instandhaltungsmaßnahmen vor dem eigentlichen Fehlereintritt ab, indem sowohl der aktuelle als auch der zukünftige Anlagenzustand inklusive der Umgebungsbedingungen diagnostiziert bzw. prognostiziert werden. Dessen Einführung bedarf allerdings einen deutlich höheren Digitalisierungsgrad und ist daher mit kostenintensiven Investitionen verbunden. Insofern ist PdM nicht für jede Anlagenklasse wirtschaftlich und prozessual sinnvoll. Der zentrale Aspekt, ob der Entwicklungs- und Datenerhebungsaufwand durch Einsparungen bei einer PdM-Strategie überhaupt refinanziert werden kann, wurde von der Forschung bislang weitgehend außer Acht gelassen. Wissenschaftler haben sich stattdessen auf die Entwicklung von Verfahren zur Bestimmung des Verschleißgrads von Komponenten und damit auf die technischen Belange konzentriert. Vor diesem Hintergrund untersucht dieser Beitrag innerhalb einer multiplen explorativen Fallstudie am Flughafen München drei repräsentative Anlagenklassen von Flughäfen - Aufzüge, Pre-Conditioned-Air(PCA)-Anlagen und Multienteiser - in Bezug auf Aufwands-, Nutzen- und Risikoaspekte des PdM. Im Ergebnis zeigten sich deutliche Unterschiede zwischen den Anlagenklassen: Während Aufzüge und PCA-Anlagen hohes Potenzial aufweisen, ist die Einführung für Multienteiser aufgrund der lediglich saisonalen Nutzung wirtschaftlich nicht sinnvoll. Mit diesem Beitrag erhalten Entscheidungsträger in Unternehmen eine Orientierungshilfe, für welche Anlagenklassen PdM profitabel ist und welche Aspekte in die ökonomische Bewertung technischer Anlagen grundsätzlich einbezogen werden sollten. Das angewandte Bewertungsverfahren dient als Muster für die Analyse weiterer technischer Anlagen.

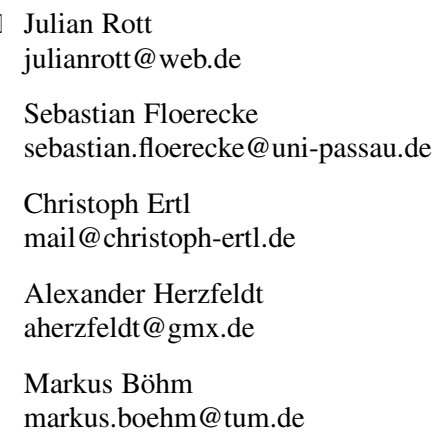

Helmut Krcmar

helmut.krcmar@tum.de

1 Lehrstuhl für Wirtschaftsinformatik und Geschäftsprozessmanagement, Technische Universität München, München, Deutschland

2 Lehrstuhl für Wirtschaftsinformatik mit Schwerpunkt Informations- und IT-Service-Management, Universität Passau, Passau, Deutschland 


\section{Einleitung}

Treten bei technischen Anlagen entlang ihres Lebenszyklus Fehler auf, ist dieser Umstand mit hoher Wahrscheinlichkeit nicht einem plötzlichen Ereignis geschuldet, sondern das Ergebnis eines schleichenden Prozesses. Neben dem altersbedingten Verschleiß beeinflussen eine Vielzahl weiterer Faktoren, wie die Materialbeschaffenheit oder bereits durchgeführte Instandhaltungsmaßnahmen das Auftreten eines technischen Fehlers. Die konkreten Auswirkungen dieser Einflussfaktoren können jedoch von Personen nicht unmittelbar erkannt werden [1, 2]. Da außerdem jede Anlage in einer anderen Umgebung unter dem Einfluss spezifischer Faktorenausprägungen betrieben wird, kann die vom Hersteller vorgegebene, vorausbestimmte Instandhaltungsstrategie nicht das wirtschaftliche Optimum bedeuten [3]. Die vorbeugende Wartung findet entweder zu früh und damit unnötig oder zu spät statt.

Predictive Maintenance (PdM) beschreibt im Gegensatz dazu eine Instandhaltungsstrategie, bei der die Durchführung von Instandhaltungsmaßnahmen vor dem eigentlichen Fehlereintritt erfolgt. Dazu werden der Anlagenzustand inklusive der Umgebungsbedingungen erfasst, evaluiert und daraus Wahrscheinlichkeiten für zukünftige Fehler berechnet [4]. Die Grundvoraussetzung dafür ist, die technischen Anlagen mit adäquater Sensorik auszustatten und Algorithmen für die Diagnose und Prognose des Anlagenzustands zu entwickeln und einzusetzen. Die Einführung einer PdMStrategie ist für Unternehmen folglich mit einer nicht zu unterschätzenden initialen Investition verbunden $[5,6]$. Insofern ist PdM nicht für jede Anlagenklasse bzw. Anlage aus wirtschaftlichen und prozessualen Gesichtspunkten vorteilhaft. Eine Einzelfallprüfung ist daher unabdingbar.

Wissenschaftler haben sich bislang auf die Entwicklung von Verfahren, sogenannte PdM-Algorithmen, zur Bestimmung des Verschleißgrads einzelner Komponenten bzw. Anlagen und somit insgesamt auf die technischen Aspekte fokussiert. Weitgehend außer Acht gelassen wurde die grundsätzliche Frage, inwieweit der Entwicklungs-, Datenerhebungs- und Datenauswertungsaufwand durch Einsparungen infolge einer PdM-Strategie überhaupt refinanziert werden kann. Um diese Forschungslücke zu verkleinern, untersucht dieser Beitrag innerhalb einer multiplen explorativen Fallstudie am Flughafen München drei sich grundlegend voneinander unterscheidende Anlagenklassen - Aufzüge, Pre-Conditioned-Air(PCA)-Anlagen und Multienteiser - in Bezug auf Aufwands-, Nutzen- und Risikoaspekte des PdM. Diese drei Anlagenklassen stehen dabei stellvertretend für die an Flughäfen eingesetzten technischen Anlagen im Bereich der Gebäude (Aufzüge), der luftverkehrsspezifischen Anlagen (PCA-Anlagen) und des Fuhrparks (Multienteiser).
Der Beitrag ist folgendermaßen aufgebaut: In Kapitel 2 werden die Grundlagen zur Instandhaltung im Allgemeinen und der Instandhaltungsart PdM im Speziellen beschrieben. Zudem werden der aktuelle Stand der Forschung und damit die Forschungslücke aufgezeigt. In Kapitel 3 wird auf grundsätzlich einsetzbare Bewertungsverfahren zur Ermittlung der wirtschaftlichen Vorteilhaftigkeit von PdM eingegangen und daraus das geeignetste ausgewählt. Kapitel 4 beschreibt das Forschungsdesign und Kapitel 5 die drei betrachteten Anlagenklassen sowie deren derzeitigen Instandhaltungsprozess. Kapitel 6 präsentiert die fallspezifischen und fallübergreifenden Ergebnisse der Untersuchung. Seinen Abschluss findet dieser Beitrag mit dem Nutzen für die Wissenschaft und die betriebliche Praxis, Limitationen und weiterem Forschungsbedarf in Kapitel 7.

\section{Hintergrund und Stand der Forschung}

Der Begriff Instandhaltung beschreibt nach DIN 13306 die „Kombination aller technischen und administrativen Maßnahmen sowie Maßnahmen des Managements während des Lebenszyklus eines Objekts, die dem Erhalt oder der Wiederherstellung seines funktionsfähigen Zustands dient, sodass es die erforderte Funktion erfüllen kann“ [7]. Es werden mehrere Instandhaltungsstrategien (Abb. 1) unterschieden, von denen in Unternehmen die korrektive und die vorausbestimmte Instandhaltung am weitesten verbreitet sind [8].

Bei der korrektiven Instandhaltung erfolgt die Instandhaltungsmaßnahme nach dem Auftreten eines Fehlers. Die Entscheidung zur Durchführung einer aktiven Instandhaltung wird somit durch den Fehler der Anlage selbst hervorgerufen [5, 7]. Die vorausbestimmte Instandhaltung wird dagegen in regelmäßigen Zeitabständen ausgeführt. Bei beiden Formen bietet ein höherer Digitalisierungsgrad zur Entscheidungsunterstützung keinerlei Vorteile. Dies steht im Gegensatz zur zustandsorientierten Instandhaltung, dem Predictive Maintenance (PdM), dessen Verbreitung durch die gestiegenen Möglichkeiten moderner Informations- und Kommunikationstechnik ausgelöst wurde [6, 8]. Ergänzend ist zu betonen, dass keine allgemein akzeptierte Definition für PdM in der Literatur existiert [8] und im englischen Sprachraum die Begriffe Condition-based Maintenance $(\mathrm{CbM})$ und PdM entweder gleichgesetzt oder anhand der voraussagenden $(\mathrm{PdM})$ und nichtvoraussagenden $(\mathrm{CbM})$ Form unterschieden werden. Für den weiteren Verlauf dieses Beitrags wird die breitere Begriffsdefinition gewählt und die voraussagende und nichtvoraussagende zustandsorientierte Instandhaltung als Reifegrade des PdM interpretiert [6].

Basierend auf einer seitens der Autoren dieses Beitrags durchgeführten strukturierten Literaturrecherche wur- 
Abb. 1 Instandhaltungsstrategien [7]

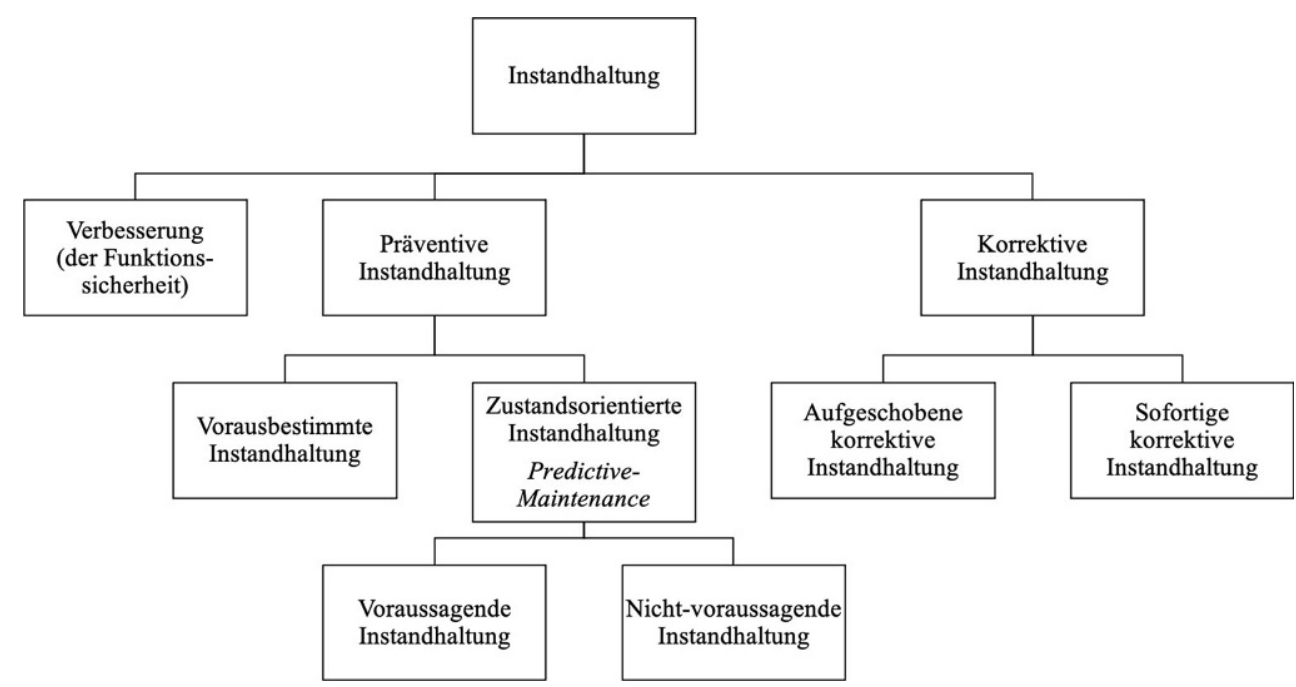

den die einzelnen Dimensionen von PdM (Konfiguration, Zustandsmonitoring, Datenakquise und -konvertierung, Diagnose und Prognose), die im praktischen Kontext implementiert werden müssen, ermittelt:

Innerhalb der (1) Konfiguration (Abb. 2) wird festgelegt, wie viele Anlagen und Komponenten die PdM-Strategie umfassen soll, in welchen zeitlichen Abständen Daten erhoben werden und welche Fehlerarten analysiert bzw. prognostiziert werden. Zudem werden die Art und die Anzahl der Instandhaltungsmaßnahmen konkretisiert und entschieden, nach welchen Kriterien die PdM-Strategie optimiert wird.

Das (2) Zustandsmonitoring dient der Erfassung des aktuellen Anlagenzustands und setzt sich aus zwei Phasen zusammen: (2a) Datenakquise und (2b) Datenkonvertierung. Erstere umfasst sämtliche Verfahren zur Erhebung von Event- und Zustandsdaten. Unter Eventdaten werden Informationen über vergangene Ereignisse verstanden. Sie geben Aufschluss darüber, was vorgefallen ist (Beispiel: Ausfall), was die Ursache war (Beispiel: Kurzschluss) und welche Maßnahmen daraufhin ausgelöst wurden (Beispiel: Komponententausch) [9]. Zustandsdaten beschreiben den aktuellen Zustand einer Anlage. Diese werden für gewöhnlich durch verschiedenste Sensoren generiert, welche die Daten automatisch erfassen und dadurch eine kontinuierliche Beobachtung des Anlagenzustands ermöglichen [9]. Um anhand der erfassten Daten das Verschleißverhalten des untersuchten Objekts zu beschreiben, existieren verschiedene Datenkonvertierungsmodelle, welche die spätere Entscheidungsfindung zur Durchführung einer Instandhaltungsmaßnahme im PdM-Prozess maßgeblich beeinflussen [10] und daher den zentralen Teil einer PdM-Strategie bilden [11]. Im Wesentlichen kann zwischen stochastischen Modellen, wie dem Gamma- oder dem Markov-Prozess, und nichtstochastischen Modellen, wie einem Expertenoder einem regelbasierten System, unterschieden werden. Für detaillierte Informationen zu den einzelnen Modellen wird insbesondere auf $[9,12]$ und [13] verwiesen.

Nach der Datenkonvertierung können die (3) Diagnose und die (4) Prognose des Anlagenzustands erfolgen. Bei
Element

\begin{tabular}{|c|}
\hline Anlagenanzahl \\
\hline Komponentenanzahl \\
\hline Art der Observierung \\
\hline Zeitperioden der Observierung \\
\hline Betriebsstatus bei Observierung \\
\hline Fehlerart \\
\hline Maschinenzustände \\
\hline
\end{tabular}

Art der Instandhaltungsmaßnahme

Anzahl der Instandhaltungsmaßnahmen

Optimierungskriterium

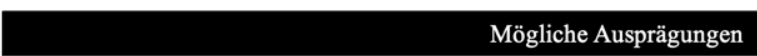

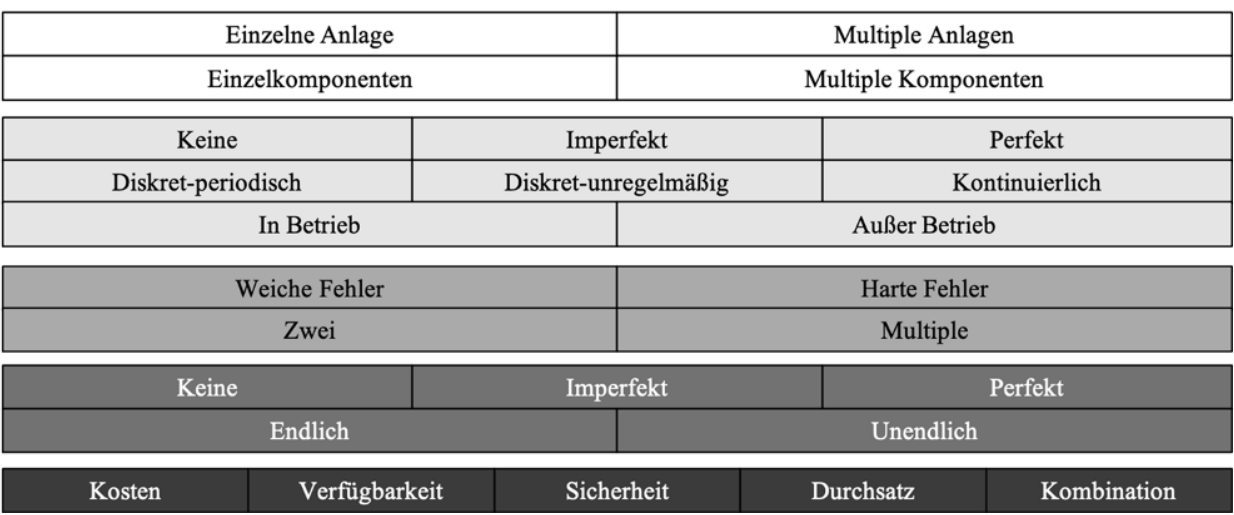

Abb. 2 Aus der Literatur abgeleitete Konfigurationsmöglichkeiten des PdM (Predictive Maintenance) 
ersterer werden historische Informationen [14] mit dem Ziel analysiert, den Fehlerursprung zu identifizieren [5, 9, 15] und ein Frühwarnsystem zu etablieren, das den Verantwortlichen Hinweise auf ein abnormales Verhalten der Anlage liefert. Der Fokus der Prognose liegt auf der Evaluation des zukünftigen Anlagenzustands und somit auf einer vorzeitigen Eventanalyse. Hierdurch verbleibt ein größerer Zeitraum für das Definieren und Planen von Instandhaltungsmaßnahmen [16].

Bisherige Veröffentlichungen zu PdM haben überwiegend eigene PdM-Algorithmen für eine bestimmte Anlagenklasse entwickelt und im Nachgang die monetäre Vorteilhaftigkeit anhand eines konstruierten bzw. realen Anwendungsfalls bewertet. Beispiele hierfür sind die Analyse von Eisenbahnschienen [17], Galliumarsenid-Laser [18], Helikoptergetrieben [19] und Generatoren [20]. Die jeweils ermittelten Einsparpotenziale weichen deutlich voneinander ab: Während etwa Sharma et al. [17] eine 10\%ige Reduktion simulieren, können die Instandhaltungskosten in der Studie von Yildirim et al. [20] um 55\% gesenkt werden. Wie viel Aufwand in die Entwicklung und Datenerfassung für PdM gesteckt wurde und welcher Gesamtnutzen somit aus PdM resultiert, hat keine Studie adressiert.

Im Unterschied zu den existierenden Forschungsarbeiten verzichtet dieser Beitrag auf die Entwicklung eines PdMAlgorithmus. Denn im praktischen Kontext erfolgt eine Entscheidung für oder wider die Implementierung einer PdMStrategie vor der eigentlichen Investition in Soft- und Hardware und somit ebenfalls vor der Entwicklung eines entsprechenden Algorithmus. Daher werden in diesem Beitrag quantitative und qualitative Aspekte, die aus der Einführung einer PdM-Strategie resultieren, untersucht. Daraus lässt sich ein umfassendes Bild über deren Vorteilhaftigkeit verschaffen. Durch die Anwendung derselben Bewertungssystematik über mehrere Anlagenklassen hinweg wird obendrein aufgezeigt, für welche der betrachteten Anlagenklassen die Einführung am ehesten geeignet ist, welche Faktoren dafür ausschlaggebend sind und somit für die Analyse weiterer Anlagen verwendet werden können.

\section{Verfahren zur Evaluation von PdM- Strategien}

Für die Durchführung dieser Studie war es notwendig, vorab ein Bewertungsverfahren auszuwählen, das die Evaluation sowohl der qualitativen als auch der quantitativen Auswirkungen einer PdM-Strategie beinhaltet. Vor diesem Hintergrund werden nachfolgend generische sowie explizit Digitalisierungsbezug aufweisende Bewertungsverfahren auf ihre diesbezügliche Eignung hin analysiert.

\section{Generische Bewertungsverfahren}

Mithilfe der Investitionsrechnung lassen sich die finanziellen Auswirkungen von Investitionsvorhaben ermitteln. Grundsätzlich wird unterschieden, ob ein repräsentatives Durchschnittsjahr (statisch) oder mehrere Perioden (dynamisch) einbezogen werden. Ein weiteres Unterscheidungsmerkmal ist, ob ausschließlich quantitative Aspekte (eindimensional) oder zusätzlich qualitative Faktoren (mehrdimensional) betrachtet werden [21]. Da die Einführung einer PdM-Strategie mit einer Investition, insbesondere in Software und Sensorik, verbunden ist, lassen sich die traditionellen Verfahren der Investitionsrechnung grundsätzlich anwenden. Allerdings ist die Anwendung einer einzigen generischen Methode für die umfassende Evaluation im PdM-Kontext nicht ausreichend [6]. Dies liegt zum einen daran, dass die eindimensionalen Methoden eine ausschließlich quantitative Bewertung vornehmen und wichtige qualitative Aspekte, wie beispielsweise die Anlagenverfügbarkeit außen, vorlassen. Zum anderen haben die meisten mehrdimensionalen Verfahren gemeinsam, dass unterschiedliche Investitionsalternativen miteinander verglichen werden, die bei der Evaluation von PdM nicht zwangsläufig vorliegen. Vielmehr geht es um den Vergleich des Ist-Zustands mit einem möglichen PdM-Prozess, um eine Aussage über die Vorteilhaftigkeit von PdM treffen zu können.

\section{Bewertungsverfahren mit Digitalisierungsbezug}

In Anbetracht der lediglich rudimentären Eignung der generischen Bewertungsverfahren für PdM wurde eine systematische Literaturrecherche [22] nach Bewertungsmodellen mit expliziten Digitalisierungsbezug durchgeführt. Hierbei wurden mittels nachfolgender Suchwörter die führenden Literaturdatenbanken EBSCO Host, Google Scholar, Science Direct und Scopus durchsucht: (digitization OR digital innovation $O R$ digital transformation) AND (evaluation $O R$ assessment) AND (method OR model) AND (process OR economic). Anhand des Titels, der Zusammenfassung und der Schlüsselwörter wurden (nach erfolgter Vorwärts- und Rückwärtssuche) insgesamt 16 Publikationen mit 15 verschiedenen Bewertungsmodellen (zwei Publikationen betrachten das gleiche Bewertungsmodell) herausgefiltert, die zusammen mit dem einzigen, speziell für PdM entwickelten Verfahrensmodell von Tauterat [6] analysiert wurden. Als relevant wurden Publikationen eingestuft, die eine eigene Bewertungssystematik für die digitale Branche vorschlagen.

Da dieser Beitrag verschiedene technische Anlagen auf Basis der Instandhaltungsprozesse miteinander vergleicht, stellen Geschäftsprozesse das Bewertungsobjekt des Verfahrens dar. Daher wurden diejenigen Methoden ausge- 
Tab. 1 Gegenüberstellung der Bewertungsverfahren der Geschäftsprozessebene für Predictive Maintenance (PdM)

\begin{tabular}{|c|c|c|c|c|}
\hline Name & DREAMY [24, 25] & Technology Map [26] & PriMa-X [23] & $\begin{array}{l}\text { PdM-Verfahrensmodell } \\
\text { [6] }\end{array}$ \\
\hline Vorgehen & $\begin{array}{l}\text { Beantwortung eines } \\
\text { Fragenkatalogs }\end{array}$ & $\begin{array}{l}\text { Angabe von Anwendungsszenario, } \\
\text { Produktlebenszyklusphase und Mitar- } \\
\text { beiterziele }\end{array}$ & $\begin{array}{l}\text { Iterative Analyse der Be- } \\
\text { standteile einer Prescriptive- } \\
\text { Maintenance-Strategie }\end{array}$ & $\begin{array}{l}\text { Bewertung von } \\
\text { Aufwands-, Nutzen- } \\
\text { und Risikoaspekten des } \\
\text { PdM }\end{array}$ \\
\hline Ziel & $\begin{array}{l}\text { Entwicklung einer } \\
\text { Roadmap zur digi- } \\
\text { talen Transformation }\end{array}$ & $\begin{array}{l}\text { Ermittlung relevanter Technologien } \\
\text { für ein unternehmensspezifisches } \\
\text { Problem }\end{array}$ & $\begin{array}{l}\text { Konfiguration des } \\
\text { Prescriptive-Maintenance- } \\
\text { Prozesses }\end{array}$ & $\begin{array}{l}\text { Qualitative und quanti- } \\
\text { tative Bewertung einer } \\
\text { PdM-Strategie }\end{array}$ \\
\hline \multicolumn{5}{|c|}{ Anforderungen/Bewertung } \\
\hline $\begin{array}{l}\text { Bewertungs- } \\
\text { objekt }\end{array}$ & Geschäftsprozess & Anwendungsszenario & Instandhaltungsprozess & Instandhaltungsprozess \\
\hline $\begin{array}{l}\text { Bewertungs- } \\
\text { variation } \\
\text { möglich? }\end{array}$ & $\mathrm{Ja}$ & $\begin{array}{l}\text { Ja (bezogen auf die vorgeschlagenen } \\
\text { Lösungen) } \\
\text { Nein (bezogen auf Variationen inner- } \\
\text { halb der Lösungen) }\end{array}$ & $\mathrm{Ja}$ & $\mathrm{Ja}$ \\
\hline $\begin{array}{l}\text { Art der Be- } \\
\text { wertung }\end{array}$ & Qualitativ & Qualitativ & Qualitativ & $\begin{array}{l}\text { Qualitativ und } \\
\text { quantitativ }\end{array}$ \\
\hline
\end{tabular}

schlossen, die anstelle der Geschäftsprozesse die Geschäftsstrategie (4), die Geschäftsmodelle (3), die Geschäftsfähigkeiten (1), die Organisation (1), das Management (1) bzw. zugrunde liegende Daten (1) und Informationen (1) eines Unternehmens bewerten.

Somit verbleiben vier der insgesamt 16 ursprünglich identifizierten Verfahren für die Durchführung der Bewertung von Instandhaltungsprozessen. Tab. 1 stellt die vier Verfahren der Geschäftsprozessebene überblicksmäßig gegenüber:

Damit das Bewertungsmodell für die Zielstellung dieses Beitrags verwendet werden kann, müssen aus Sicht der Autoren die folgenden drei Anforderungen (A) erfüllt sein:

1. A1 Die Effekte aus der Einführung von PdM, insbesondere auf den Instandhaltungsprozess, müssen das Bewertungsobjekt des Verfahrens bilden.

2. A2 Mit dem Bewertungsverfahren muss es möglich sein, die einzelnen Anlagenklassen unterschiedlich zu bewerten, um aus dem Vergleich der Einzelbewertungen die Anlage mit dem höchsten wirtschaftlichen Potenzial ermitteln zu können.

3. A3 Neben dem wirtschaftlichen Potenzial (quantitative Bewertung) müssen für ein umfassendes Bild auch qualitative Aspekte berücksichtigt werden können.

Die Technology Map [26] schlägt zu einem gegebenen Problem mögliche Lösungen - beispielsweise PdM - vor und bietet somit eine Vorselektion geeigneter Instandhaltungsprozesse. Da jedoch keine Detaillierung, sondern lediglich eine allgemeine qualitative Bewertung stattfindet, eignet sich dieses Verfahren nicht für die (quantitative) Bewertung des Potenzials unterschiedlicher Prozesse (A2 und A3).
Auch beim Bewertungsverfahren DREAMY [24, 25] kann nur ein Hinweis über die mögliche Eignung abgegeben werden, indem der aktuelle Instandhaltungsprozess anhand eines Fragenkatalogs in ein qualitatives Reifegradmodell eingeordnet wird. Eine quantitative Untersuchung findet nicht statt (A3).

Ziel des Modells von Nemeth et al. [23] ist nicht die Auswahl einer Anlage, sondern die Konfiguration des Prescriptive Maintenance - eine Instandhaltungsstrategie, bei der Events (Beispiele: Ausfall oder Instandhaltung einer Anlage) gezielt ausgelöst werden - für ein definiertes technisches Objekt. Es lässt sich daher ebenfalls nicht für die Bewertung von unterschiedlichen Instandhaltungsprozessen einsetzen (A1, A2 und A3).

Das Verfahren von Tauterat [6] kombiniert zwei generische Bewertungsmodelle: (1) die Kostenvergleichsrechnung für die Betrachtung von Aufwandsaspekten und (2) die Prioritätenanalyse für die Evaluierung von Nutzen- und Risikofaktoren. Für den Vergleich des Ist-Prozesses mit einem möglichen PdM-Prozess wird ersterer zunächst in ein Reifegradmodell eingeordnet (Abb. 3).

Für die Aufwands-, Nutzen- und Risikobewertung stellt Tauterat [6] eine Sammlung von Nutzen- sowie Risikogruppen und -aspekten zur Verfügung. Sie kann gefiltert und um weitere ergänzt werden. Die eigentliche Bewertung beinhaltet eine Zuordnung der Aspekte zur jeweiligen Reifegradstufe (ab welcher der Aspekt eintritt) und erfolgt innerhalb von Workshops oder Interviews mit Experten. Für die Aufwandsbewertung werden außerdem drei Szenarien (bester, mittlerer und schlechtester Fall) erstellt. Nachdem das Verfahren von Tauterat [6] als einziges alle drei Anforderungen erfüllt, wird es für die vorliegende Studie verwendet. 


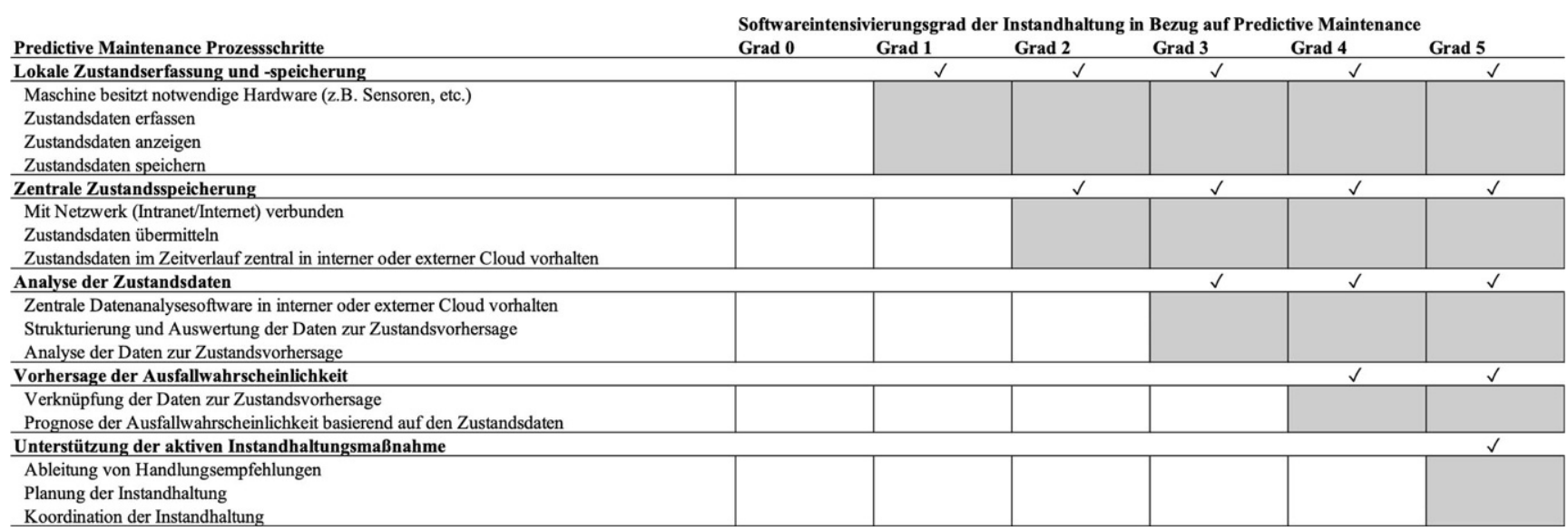

Abb. 3 PdM(Predictive Maintenance)-Reifegradmodell [6]

\section{Forschungsdesign}

Für diesen Beitrag wurde eine explorative multiple Fallstudie [27] am Flughafen München durchgeführt. Fallstudien eignen sich, um innerhalb von Gebieten mit bisher wenig durchgeführten Studien zu forschen und damit die Art und Komplexität der stattfindenden Prozesse zu verstehen sowie vom aktuellen State of the Art zu lernen und ausgehend von der Praxis Hypothesen und Theorien zu generieren [28]. Diese Kriterien treffen allesamt auf das Ziel dieses Beitrags $\mathrm{zu}$ : Die fallspezifische und fallübergreifende Untersuchung von Anlagenklassen in Bezug auf Aufwands-, Nutzen- und Risikoaspekte des PdM.

Der Betrieb des gemessen an den Passagierzahlen zweitgrößten Verkehrsflughafen Deutschlands wird von der Flughafen München GmbH (FMG) mit ihren 15 Tochtergesellschaften übernommen, die in Summe als sogenannter Full Service Operator agieren und einen Großteil der Leistungen in nahezu allen Bereichen des Flughafenmanagements selbst erbringen. Der Technikbereich der FMG ist für den Betrieb aller technischen Anlagen verantwortlich. Diese lassen sich in vier Gruppen unterteilen: (1) Fahrzeuge, (2) Gebäude, (3) Luftverkehrsflächen und -anlagen sowie (4) Anlagen der Energie-, Wasser- und Abfallwirtschaft. Aufgrund der Vielzahl und Varietät sicherheitsrelevanter, technischer Anlagen ist der Flughafen München als Forschungsobjekt für diese Studie bestens geeignet.

Um die verschiedenen Anlagentypen eines Flughafens zu repräsentieren, wurde aus den drei technischen Bereichen am Flughafen München, die PdM bislang nicht einsetzen (Fahrzeugmanagement, Luftverkehrsflächen und -anlagen und Gebäudemanagement), jeweils eine Anlage ausgewählt. Die Auswahl der Anlagen erfolgte anhand einer Kombination aus intensitäts- und kriterienbasiertem Sampling [29]. Ersteres zielt darauf ab, Fälle auszuwählen, die voraussichtlich einen hohen Informationsgehalt liefern. Das kriterienbasierte Sampling bezieht sich darauf, dass An- lagen in Eigen- und Fremdinstandhaltung mit direkt und nicht/indirekt observierbaren Stillstandskosten, von denen mehrere gleiche installiert sind, untersucht werden sollen. Die Wahl fiel schließlich auf die folgenden drei Anlagen: Multienteiser, PCA-Anlagen und Aufzüge.

Zur Sammlung von Daten, die den Ist-Prozess und die Auswirkungen einer PdM-Strategie beschreiben und somit für die Potenzialbewertung verwendet werden können, dienten in Anlehnung an [6] leitfadengestützte Workshops, in denen neben den Wortbeiträgen der Teilnehmer auch Dokumente, wie Prozessmodelle und Kostenaufstellungen, betrachtet wurden. Um eine größtmögliche Variation an Perspektiven zu erreichen, wurden der Anlagenverantwortliche, ein Mitarbeiter mit hohem Instandhaltungsbezug und der zuständige Controller zum jeweiligen Workshop eingeladen. Die drei Workshops fanden im Juni und Juli 2019 statt und dauerten je Anlage zwischen 5,5 und 6,5h. Hierbei wurden die vier Themenfelder Prozessbewertung und -einordnung sowie die Bewertung der Aufwands-, Nutzenund Risikoaspekte [6] behandelt. Die Bestimmung der Bewertungsausprägungen erfolgte innerhalb einer Gruppendiskussion.

Während der Workshops wurden handschriftliche Notizen angefertigt, aus denen im Anschluss ein inhaltlich strukturiertes Protokoll [6] erstellt und den Teilnehmern mit der Bewertung der einzelnen Aspekte zur Validierung [29] zugesandt wurde. Änderungswünsche seitens der Teilnehmer wurden im Nachgang nicht eingebracht. Bei der Analyse der Bewertungsergebnisse dienten die Protokolle insbesondere zur Interpretation der Bewertungsausprägungen. Alle Informationen und Einschätzungen in Bezug auf die jeweilige Anlage basieren somit, wenn nicht anders gekennzeichnet, auf den jeweiligen Workshops. Die Datenauswertung erfolgte auf zwei Arten: Einerseits wurde jede technische Anlage isoliert, mit dem Ziel der Beschreibung und Untersuchung des konkreten Falls, betrachtet. Andererseits fand 
im Sinne einer fallübergreifenden Analyse eine Gegenüberstellung der Ergebnisse der drei Anlagen statt [29].

\section{Beschreibung der Anlagenklassen und der gegenwärtigen Instandhaltungsprozesse}

\section{Multienteiser}

Der Flughafen München verfügt über fünf Multienteiser, die je nach Härte des Winters zwischen 50 und 400 Betriebsstunden in einer Winterdienstsaison vom 15.10.-30.04. leisten und für die Enteisung der Vorfeldflächen eingesetzt werden. Aufgrund ihrer saisonalen Nutzung werden sämtliche vorausbestimmten Instandhaltungstätigkeiten (Wartung und Unfallverhütungsvorschriften) durch eine flughafeneigene Fahrzeugwerkstatt in den Monaten Mai-September umgesetzt.

Bei der Durchführung der vorausbestimmten Instandhaltung wird neben den vom Hersteller empfohlenen Tätigkeiten eine optische Sichtkontrolle vorgenommen. Diese dient dem frühzeitigen Erkennen und dem Austausch von Komponenten, die innerhalb der bevorstehenden Winterdienstsaison ausfallen könnten bzw. mit hoher Wahrscheinlichkeit ersetzt werden müssten. Während des Winters erforderliche Instandsetzungstätigkeiten resultieren überwiegend aus Schadensvorfällen.

\section{Pre-Conditioned-Air(PCA)-Anlagen}

Um Flugzeuge auf der Parkposition mit vorklimatisierter Frischluft zu versorgen, setzt der Münchner Flughafen PreConditioned-Air(PCA)-Anlagen ein. Die PCA-Anlagen sind im Vergleich zu den eigentlich für die Klimatisierung am Flugzeug vorgesehenen Hilfsturbinen (Auxiliary Power Unit) im Betrieb deutlich leiser, senken den Kerosinverbrauch und den damit verbundenen $\mathrm{CO}_{2}$-Ausstoß. Derzeit sind zwölf dieser Anlagen am Terminal 1 installiert.

Die vorausbestimmte Instandhaltung besteht aus einer monatlichen Inspektion, einer halbjährlichen Hygienesowie einer jährlichen umfänglichen Wartung. Eine Herausforderung besteht darin, dass sich die Nutzungsintensität der einzelnen PCA-Anlagen deutlich unterscheidet, da der jeweilige Pilot selbst über die Verwendung entscheidet. Demzufolge ist der Zeitpunkt der vorausbestimmten Wartung bzw. Inspektion bei viel genutzten Anlagen tendenziell verspätet und bei selten genutzten Anlagen verfrüht. Die Störbeseitigung und Instandsetzung erfolgen nach dem Auftreten von Fehlern oder dem Ausfall von Komponenten (korrektive Instandhaltung).

\section{Aufzugsanlagen}

Am Flughafen München sind rund 300 Aufzüge in Betrieb. Im Gegensatz zu den beiden anderen Anlagenklassen befinden sich häufig mehrere Aufzüge in unmittelbarer Nachbarschaft. Dies liegt zum einen daran, dass die Aufzüge gegenseitig als Rückfallstufe dienen. Zum anderen sind mancherorts mehrere Anlagen zur Bewältigung der Spitzenlast nötig.

Die Aufzüge werden durch beauftragte Dienstleister bzw. die Hersteller instand gehalten. Die Vergütung erfolgt im Rahmen eines Dienstvertrags anhand einer jährlichen Pauschale je Aufzug. Der Vertragspartner ist dabei für die Durchführung aller Instandhaltungstätigkeiten - mit Ausnahme von Vandalismusvorfällen - verantwortlich. Die Instandhaltung geschieht demnach nach den Prinzipien der vorausbestimmten und korrektiven Ausprägung.

\section{Wirtschaftliche Evaluation der drei Anlagen}

Anhand des Verfahrensmodells von Tauterat [6] wird in diesem Kapitel nacheinander auf die Aufwands-, Nutzen- und Risikoaspekte eingegangen, die aus der PdM-Einführung für die drei Anlagenklassen resultieren. Die darauffolgende Gesamteinschätzung fasst die wesentlichen Punkte der einzelnen Aspekte anlagenvergleichend zusammen.

\section{Aufwandsanalyse}

\section{Kosteneinsparung durch PdM}

Da die korrektive Instandhaltung der Multienteiser nahezu ausschließlich aus Fremdeinwirkungen resultiert und die vorausbestimmte Instandhaltung bereits erfahrungsbasiert optimiert durchgeführt wird, ist keine Auswirkung von PdM auf die Instandhaltungskosten zu erwarten.

Anders verhält es sich bei den Aufzugs- und PCA-Anlagen. In Tab. 2 sind die erwarteten Auswirkungen (+: Zusatzkosten; -: Kosteneinsparung) einer PdM-Einführung - unterschieden nach drei Szenarien (schlechtester, mittlerer und bester Fall) - aufgeführt:

Für die meisten Instandhaltungstätigkeiten zeigt sich eine deutlich höhere erwartete Einsparung bei den Aufzugsals bei den PCA-Anlagen. Lediglich bei der Instandsetzung wird von einer größeren relativen Einsparung bei den PCA-Anlagen ausgegangen. Die Ergebnisse hinsichtlich der Kosteneinsparungen lassen sich wie folgt begründen: Zum einen erfolgt die Instandhaltung der Aufzüge durch eine Fremdfirma über Pauschalen und nicht, wie bei den PCA-Anlagen, durch eigene Mitarbeiter. Um das Verlustrisiko aufseiten der Instandhaltungsfirma zu verringern, ist in der Kalkulation ein Zuschlag enthalten. Zum anderen 
Tab. 2 Kosteneinsparung durch Predictive Maintenance bei PCA(Pre Conditioned Air)-Anlagen und Aufzügen

\begin{tabular}{|c|c|c|c|c|c|c|c|c|}
\hline \multirow[b]{2}{*}{ Instandhaltungstätigkeiten } & \multicolumn{2}{|l|}{ Status quo } & \multicolumn{2}{|c|}{ Schlechtester Fall } & \multicolumn{2}{|c|}{ Mittlerer Fall } & \multicolumn{2}{|l|}{ Bester Fall } \\
\hline & $\begin{array}{l}\text { Aufzüge in } \\
\%\end{array}$ & $\begin{array}{l}\text { PCA in } \\
\%\end{array}$ & $\begin{array}{l}\text { Aufzüge in } \\
\%\end{array}$ & PCA in $\%$ & $\begin{array}{l}\text { Aufzüge in } \\
\%\end{array}$ & $\begin{array}{l}\text { PCA in } \\
\%\end{array}$ & $\begin{array}{l}\text { Aufzüge in } \\
\%\end{array}$ & $\begin{array}{l}\text { PCA in } \\
\%\end{array}$ \\
\hline Inspektion & 10,0 & 15,0 & 0,0 & 6,7 & $-25,0$ & 0,0 & $-50,0$ & $-10,0$ \\
\hline Wartung & 40,0 & 40,0 & $-10,0$ & 5,0 & $-33,3$ & $-5,0$ & $-50,0$ & $-15,0$ \\
\hline Störbeseitigung & 20,0 & 20,0 & $-10,0$ & 0,0 & $-42,5$ & 0,0 & $-75,0$ & 0,0 \\
\hline Instandsetzung & 30,0 & 25,0 & 0,0 & 0,0 & 0,0 & $-10,0$ & $-16,7$ & $-20,0$ \\
\hline Summe & 100,0 & 100,0 & 94,0 & 103,0 & 75,7 & 95,5 & 55,0 & 87,5 \\
\hline
\end{tabular}

ist an einem Aufzug bereits ein PdM-Prototyp installiert. Da somit bereits Erfahrungen mit PdM bei Aufzügen gesammelt werden konnten, ist davon auszugehen, dass das tiefere Verständnis der Thematik zu höheren Prognosen in Bezug auf das wirtschaftliche Potenzial führt.

\section{Kosten des PdM-Systems}

Die in Abb. 4 dargestellte Kostenschätzung (absolut und relativ im Vergleich zu den jährlichen Instandhaltungskosten) basiert für die Aufzugsanlagen auf einer Hochrechnung eines vorliegenden Angebots zur Prototypeninstallation. Da für Multienteiser und PCA-Anlagen nach Kenntnis der Autoren kein PdM-System auf dem Markt verfügbar ist, wurde hierfür eine eigene Kostenschätzung angestellt, die auf den Einsichten der Workshops und Erfahrungen aus einem PdM-Projekt am Flughafen München basiert. Folglich weist die Kostenprognose für Multienteiser und PCA-Anlagen eine höhere Unsicherheit auf.

Aus der Gegenüberstellung der Kostenprognosen geht hervor, dass die Installation von PdM bei einer Vielzahl von Anlagen mit einer hohen initialen Investition in Hardware einhergeht. Während für die Aufzugsanlagen die einmaligen Aufwendungen vordergründig den Erwerb und die Installation der Hardwarekomponenten umfassen, machen bei den PCA-Anlagen und den Multienteisern die Softwareentwicklungskosten den Hauptanteil aus. Nachdem am Markt derzeit keine Standardlösung für PdM bei Multienteisern und PCA-Anlagen verfügbar ist, muss hierfür eigens ein spezifischer PdM-Algorithmus entwickelt werden.

\section{Nutzenanalyse}

\section{Relevanzhäufigkeit}

Wie aus Tab. 3 hervorgeht, steht bei der Einführung von PdM bei allen drei Anlagen die Erhöhung der Verfügbarkeit und die Optimierung des Personaleinsatzes im Vordergrund.

Als zentrales Ergebnis lässt sich festhalten: Alle Nutzengruppen wurden mindestens bei einer Anlage als relevant eingestuft. Dass elf der insgesamt 17 Nutzengruppen lediglich bei einer der drei Anlagen von Bedeutung sind, kann als Indiz dafür gewertet werden, dass die Ziele von PdM stark von den Besonderheiten einer Anlagenklasse und dem jeweiligen Kontext abhängen. Beispielsweise ist die verbesserte Steuerung des Fremddienstleisters nur für die Aufzugsanlagen relevant, da diese Gegenstand eines Vollinstandhaltungsvertrags sind und somit nicht von der FMG selbst instand gehalten werden. Ähnliches gilt für „Redundantes und/oder Reservegerät optimieren“. Denn nur bei den Aufzugsanlagen sind für gewöhnlich mindestens zwei redundante Anlagen installiert.

\section{Reifegradeinordnung}

Aus der Gegenüberstellung der Reifegradeinordnungen der Nutzenaspekte aller drei Anlagen (Tab. 4), ab welchem Reifegrad der Nutzen eintritt, wird sichtbar, dass die Mehrzahl an Nutzenaspekten (21 von 36) mit der Implementierung eines PdM-Systems der dritten Stufe (Zustandsdatenanalyse) realisiert wird. Dass dies nicht der Einordnung einer spezifischen Anlage geschuldet ist, zeigt sich darin, dass für jede der drei untersuchten Anlagen bei der dritten Stufe die meisten Nutzenaspekte ausgelöst werden.

Außerdem wird deutlich, dass ausschließlich bei den Multienteisern die lokale und bei den PCA-Anlagen die
Anzahl der Anlagen Installation, Schulung \& Entwicklung (einmalig) Kosten PdM Hardware (einmalig) Software (laufend)

\begin{tabular}{cc}
\multicolumn{2}{c}{ Multienteiser } \\
\multicolumn{2}{c}{5} \\
$271.000 €$ & $366 \%$ \\
$12.500 €$ & $17 \%$ \\
$2.000 €$ & $3 \%$
\end{tabular}

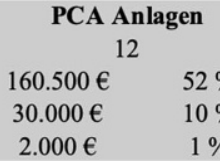

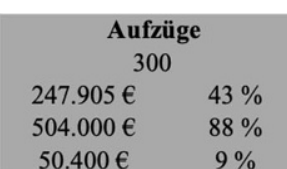

Abb. 4 Vergleich der Kosten aus der Predictive-Maintenance-Einführung. PCA Pre Conditioned Air 
Tab. 3 Relevanzhäufigkeit der Nutzengruppen bei der Predictive-Maintenance-Einführung

\begin{tabular}{|c|c|c|c|}
\hline $\begin{array}{l}\text { Bei keiner Anlage } \\
\text { relevant }\end{array}$ & Bei einer von drei Anlagen relevant & $\begin{array}{l}\text { Bei zwei von drei Anlagen } \\
\text { relevant }\end{array}$ & Bei allen Anlagen relevant \\
\hline \multirow[t]{11}{*}{$\overline{-}$} & Produktqualität $(\mathrm{P})$ & Kosteneinsparung $(\mathrm{A}, \mathrm{P})$ & $\begin{array}{l}\text { Anlagen-, Maschinen- und Bauteilverfüg- } \\
\text { barkeit (A, M, P) }\end{array}$ \\
\hline & Umsatzerhöhung (P) & $\begin{array}{l}\text { Prozess- und Produktionsqua- } \\
\text { lität }(\mathrm{M}, \mathrm{P})\end{array}$ & Personaleinsatz $(\mathrm{A}, \mathrm{M}, \mathrm{P})$ \\
\hline & Allgemein $(\mathrm{P})$ & Planungssicherheit $(\mathrm{A}, \mathrm{P})$ & \\
\hline & $\begin{array}{l}\text { Nachhaltigkeit (APU-Nutzung nicht } \\
\text { notwendig) (P) }\end{array}$ & $\begin{array}{l}\text { Stillstandszeiten } \\
\text { optimieren }(A, P)\end{array}$ & \\
\hline & Sicherheit $(\mathrm{M})$ & & \\
\hline & $\begin{array}{l}\text { Steuerung des Fremddienstleis- } \\
\text { ters (A) }\end{array}$ & & \\
\hline & Transparenz (A) & & \\
\hline & Kunden (A) & & \\
\hline & Ersatzteilplanung verbessern (A) & & \\
\hline & Zusätzliche Services (A) & & \\
\hline & $\begin{array}{l}\text { Redundantes und/oder Reservegerät } \\
\text { optimieren (A) }\end{array}$ & & \\
\hline
\end{tabular}

$A$ Aufzüge, $A P U$ Auxiliary Power Unit, $M$ Multienteiser, $P$ Pre-Conditioned-Air-Anlagen

Tab. 4 Vergleich der Reifegradeinordnung der Nutzenaspekte

\begin{tabular}{|c|c|c|c|c|c|c|c|c|c|}
\hline \multirow[t]{2}{*}{ Reifegrad } & \multirow[t]{2}{*}{ Nutzenaspekte } & \multicolumn{2}{|c|}{ Multienteiser } & \multicolumn{2}{|c|}{$\begin{array}{l}\text { Pre-Conditioned-Air- } \\
\text { Anlagen }\end{array}$} & \multicolumn{2}{|l|}{ Aufzüge } & \multicolumn{2}{|l|}{ Summe } \\
\hline & & $\begin{array}{l}\text { Einzelbe- } \\
\text { trachtung }\end{array}$ & Kumuliert & $\begin{array}{l}\text { Einzelbe- } \\
\text { trachtung }\end{array}$ & Kumuliert & $\begin{array}{l}\text { Einzel- } \\
\text { betrachtung }\end{array}$ & Kumuliert & $\begin{array}{l}\text { Einzel- } \\
\text { betrachtung }\end{array}$ & $\begin{array}{l}\text { Kumu- } \\
\text { liert }\end{array}$ \\
\hline 1 & $\begin{array}{l}\text { Lokale Zustandserfas- } \\
\text { sung und -speicherung }\end{array}$ & 1 & 1 & 0 & 0 & 0 & 0 & 1 & 1 \\
\hline 2 & $\begin{array}{l}\text { Zentrale Zu- } \\
\text { standsspeicherung }\end{array}$ & 0 & 1 & 7 & 7 & 0 & 0 & 7 & 8 \\
\hline 3 & $\begin{array}{l}\text { Analyse der Zustands- } \\
\text { daten }\end{array}$ & 3 & 4 & 7 & 14 & 11 & 11 & 21 & 29 \\
\hline 4 & $\begin{array}{l}\text { Vorhersage der Aus- } \\
\text { fallwahrscheinlichkeit }\end{array}$ & 0 & 4 & 2 & 16 & 1 & 12 & 3 & 32 \\
\hline 5 & $\begin{array}{l}\text { Unterstützung der } \\
\text { aktiven Instandhal- } \\
\text { tungsmaßnahme }\end{array}$ & 1 & 5 & 0 & 16 & 3 & 15 & 4 & 36 \\
\hline
\end{tabular}

zentrale Zustandsspeicherung zusätzlichen Nutzen generiert. Zurückzuführen lässt sich dieser Umstand bei den PCA-Anlagen darauf, dass durch die zentrale Transparenz über aktuelle Zustandsdaten manuelle Prüfungen vor Ort reduziert werden können. Da bei den Multienteisern aktuell keine Zustandsdaten vorliegen, führt schon die lokale Erfassung zur Generierung von Erfahrungswissen, das für zukünftige Instandhaltungen verwendet werden kann.

\section{Risikoanalyse}

\section{Relevanzhäufigkeit}

Bei der Betrachtung der als relevant identifizierten Risikogruppen (Tab. 5) wird der Vorteil der Eigentümerschaft an den Anlagen deutlich: Bei keiner der drei untersuchten
Anlagen wird die Datenbereitstellung für PdM als Risiko eingestuft.

Hingegen stellen die Kosten für alle drei Anlagenklassen ein relevantes Risiko dar. Dies bezieht sich sowohl auf die Kostenprognose des PdM, also die Kosten, die aus der Einführung von PdM resultieren, als auch auf die erwartete Kosteneinsparung durch die geänderte Instandhaltungsstrategie. Als weiteres für alle drei Anlagen zutreffendes Risiko gilt die Daten- und Voraussagequalität. Insbesondere wenn die Vorhersagen des PdM automatisiert Entscheidungen auslösen, wie etwa die Terminierung einer Instandhaltungsmaßnahme oder die Bestellung eines Ersatzteils, müssen die zugrunde liegenden Daten sowie das Vorhersagemodell eine hohe Qualität aufweisen.

Die Risiken des PdM-Systems (erhöhte Störanfälligkeit, nicht beherrschbare Komplexität und zu große Datenmen- 
Tab. 5 Relevanzhäufigkeit der Risikogruppen bei der PdM(Predictive Maintenance)-Einführung

\begin{tabular}{llll}
\hline $\begin{array}{l}\text { Bei keiner Anlage } \\
\text { relevant }\end{array}$ & $\begin{array}{l}\text { Bei einer von drei Anlagen } \\
\text { relevant }\end{array}$ & $\begin{array}{l}\text { Bei zwei von drei Anlagen } \\
\text { relevant }\end{array}$ & Bei allen drei Anlagen relevant \\
\hline Datenbereitstellung & $\begin{array}{l}\text { Risiko für das } \\
\text { Unternehmen (M) }\end{array}$ & Datensicherheit (A, P) & Daten- und Voraussagequalität (A, M, P) \\
& Risiken des PdM-Systems (M, P) & Kosten (A, M, P) \\
\hline
\end{tabular}

$A$ Aufzüge, $M$ Multienteiser, $P$ Pre-Conditioned-Air-Anlagen

Tab. 6 Vergleich der Reifegradeinordnung der Risikoaspekte

\begin{tabular}{|c|c|c|c|c|c|c|c|c|c|}
\hline \multirow[t]{2}{*}{ Reifegrad } & \multirow[t]{2}{*}{ Risikoaspekte } & \multicolumn{2}{|c|}{ Multienteiser } & \multicolumn{2}{|c|}{$\begin{array}{l}\text { Pre-Conditioned-Air- } \\
\text { Anlagen }\end{array}$} & \multicolumn{2}{|l|}{ Aufzüge } & \multicolumn{2}{|l|}{ Summe } \\
\hline & & $\begin{array}{l}\text { Einzelbe- } \\
\text { trachtung }\end{array}$ & Kumuliert & $\begin{array}{l}\text { Einzelbe- } \\
\text { trachtung }\end{array}$ & Kumuliert & $\begin{array}{l}\text { Einzelbe- } \\
\text { trachtung }\end{array}$ & Kumuliert & $\begin{array}{l}\text { Einzelbe- } \\
\text { trachtung }\end{array}$ & $\begin{array}{l}\text { Kumu- } \\
\text { liert }\end{array}$ \\
\hline 1 & $\begin{array}{l}\text { Lokale Zustandserfas- } \\
\text { sung und -speicherung }\end{array}$ & 3 & 3 & 2 & 2 & 0 & 0 & 5 & 5 \\
\hline 2 & $\begin{array}{l}\text { Zentrale Zu- } \\
\text { standsspeicherung }\end{array}$ & 1 & 4 & 1 & 3 & 1 & 1 & 3 & 8 \\
\hline 3 & $\begin{array}{l}\text { Analyse der Zustands- } \\
\text { daten }\end{array}$ & 4 & 8 & 2 & 5 & 2 & 3 & 8 & 16 \\
\hline 4 & $\begin{array}{l}\text { Vorhersage der Aus- } \\
\text { fallwahrscheinlichkeit }\end{array}$ & 1 & 9 & 1 & 6 & 1 & 4 & 3 & 19 \\
\hline 5 & $\begin{array}{l}\text { Unterstützung der } \\
\text { aktiven Instandhal- } \\
\text { tungsmaßnahme }\end{array}$ & 1 & 10 & 1 & 7 & 2 & 6 & 4 & 23 \\
\hline
\end{tabular}

gen) werden nur bei den Multienteisern und den PCA-Anlagen als Risiko eingestuft. Die Vermutung liegt nahe, dass der installierte Prototyp bei den Aufzugsanlagen zeigt, dass große Datenmengen zielgerichtet verarbeitet und komplexe Sachverhalte dargestellt werden können sowie keine erhöhte Fehleranfälligkeit aus einer Installation entsteht.

\section{Reifegradeinordnung}

Ähnlich der Einordnung der Nutzenaspekte werden relativ gesehen auch die meisten Risiken (8 von 23) durch die Analyse der Zustandsdaten ausgelöst (Tab. 6). Der Anteil ist mit $35 \%$ jedoch deutlich geringer (Nutzenaspekte: $58 \%$ ).

Weitere fünf Risiken treten bei den Multienteisern (fehlende Akzeptanz der Mitarbeiter; Vertrauen in die Daten und Software; erhöhte Fehler- und Störanfälligkeit durch zusätzliche Sensoren) und PCA-Anlagen (Datenqualität; erhöhte Fehler- und Störanfälligkeit durch zusätzliche Sensoren) bereits mit der lokalen Zustandserfassung auf. Hier ergibt sich ein großer Unterschied zu den Nutzenaspekten, bei denen lediglich ein Aspekt ab der ersten Reifegradstufe relevant ist.

\section{Fallübergreifende Analyse}

Für die abschließende Ermittlung der Anlage mit dem höchsten Potenzial aus wirtschaftlicher und prozessualer
Sicht werden in Abb. 5 alle betrachteten Themenfelder gegenübergestellt:

Die ermittelte, abgeschätzte Amortisationsdauer

$$
\left(\frac{\text { Einmalige Kosten }}{\text { Erwartete Kosteneinsparung - Laufende Kosten }}\right)
$$

stellt für die Aufzugsanlagen mit 8,16 Jahren den kürzesten Zeitraum dar. Für die Multienteiser ist aufgrund der erwarteten Kosteneinsparung von $0 \%$ keine Amortisation zu erwarten. Als einzige der drei untersuchten Anlagen entstehen bei der Nichtverfügbarkeit von PCA-Anlagen direkte „Stillstandskosten“, da die Anlage nicht zur Verrechnung an die Nutzer zur Verfügung steht. Da die erwartete relative Einsparung der Instandhaltungskosten niedriger als die der Aufzugsanlagen ist, ergibt sich für die PCA-Anlagen mit 11,57 Jahren eine höhere Amortisationsdauer. Das Verhältnis von relevanten Nutzen- zu Risikoaspekten weist mit 16/7 (PCA-Anlagen) und 15/6 (Aufzüge) einen vergleichbaren Wert auf. Für die Multienteiser entsteht mit 5/10 ein Übergewicht der Risikoaspekte.

Die Aufzugsanlagen haben somit das im Vergleich größte wirtschaftliche Potenzial (höchste relative und absolute Einsparung). Außerdem ist das Verhältnis von Nutzen- zu Risikoaspekten am größten. Aufgrund der Vielzahl an Aufzügen ist die Einführung jedoch ebenfalls mit den höchsten initialen und laufenden Kosten verbunden. 


\begin{tabular}{|c|c|c|c|c|c|c|c|}
\hline \multirow{5}{*}{ Aktuelle Instandhaltungskosten } & \multirow{3}{*}{ In Summe } & \multirow{2}{*}{\multicolumn{2}{|c|}{ Multienteiser }} & \multirow{2}{*}{\multicolumn{2}{|c|}{ PCA Anlagen }} & \multirow{2}{*}{\multicolumn{2}{|c|}{ Aufzüge }} \\
\hline & & & & & & & \\
\hline & & \multicolumn{2}{|c|}{$74.000 €$} & \multicolumn{2}{|c|}{$310.000 €$} & \multicolumn{2}{|c|}{$570.000 €$} \\
\hline & Anzahl der Anlagen & \multicolumn{2}{|c|}{5} & \multicolumn{2}{|c|}{12} & \multirow{2}{*}{\multicolumn{2}{|c|}{$\begin{array}{c}300 \\
1.900 €\end{array}$}} \\
\hline & Je Anlage & \multicolumn{2}{|c|}{$14.800 €$} & \multicolumn{2}{|c|}{$25.833 €$} & & \\
\hline \multirow{4}{*}{$\begin{array}{l}\text { Einsparung durch PdM } \\
\text { (absolut/relativ) }\end{array}$} & Schlechtester Fall (Instandhaltung) & $0 €$ & $0,0 \%$ & $9.300 €$ & $3,0 \%$ & $-34.200 €$ & $-6,0 \%$ \\
\hline & Mittlerer Fall (Instandhaltung) & $0 €$ & $0,0 \%$ & $-13.950 €$ & $-4,5 \%$ & $-136.800 €$ & $-24,0 \%$ \\
\hline & Bester Fall (Instandhaltung) & $0 €$ & $0,0 \%$ & $-38.750 €$ & $-12,5 \%$ & $-256.500 €$ & $-45,0 \%$ \\
\hline & Stillstandskosten & $0 €$ & $0,0 \%$ & $-2.000 €$ & $-0,6 \%$ & $0 €$ & $0,0 \%$ \\
\hline \multirow{3}{*}{ Kosten PdM } & Installation, Schulung \& Entwicklung (einmalig) & $271.000 €$ & $366,2 \%$ & $160.500 €$ & $51,8 \%$ & $247.905 €$ & $43,5 \%$ \\
\hline & Hardware (einmalig) & $12.500 €$ & $16,9 \%$ & $30.000 €$ & $9,7 \%$ & $504.000 €$ & $88,4 \%$ \\
\hline & Software (laufend) & $2.000 €$ & $2,7 \%$ & $2.000 €$ & $0,6 \%$ & $50.400 €$ & $8,8 \%$ \\
\hline \multirow{2}{*}{ Amortisation } & Jährliche erwartete Einsparung & \multicolumn{2}{|c|}{$0,0 \%$} & \multicolumn{2}{|c|}{$-5,3 \%$} & \multicolumn{2}{|c|}{$-25,0 \%$} \\
\hline & Amortisationsdauer (in Jahren) & \multicolumn{2}{|c|}{-} & \multicolumn{2}{|c|}{11,57} & \multicolumn{2}{|c|}{8,16} \\
\hline \multirow{4}{*}{ Nutzen } & Anzahl relevanter Nutzengruppen & \multicolumn{2}{|c|}{4} & \multicolumn{2}{|c|}{10} & \multicolumn{2}{|c|}{11} \\
\hline & Am höchsten priorisierte Nutzengruppe & \multicolumn{2}{|c|}{ Sicherheit } & \multicolumn{2}{|c|}{$\begin{array}{c}\text { Nachhaltigkeit (APU } \\
\text { Nutzung nicht notwendig) }\end{array}$} & \multicolumn{2}{|c|}{ Planungssicherheit } \\
\hline & Anzahl relevanter Nutzenaspekte & \multirow{2}{*}{\multicolumn{2}{|c|}{$\begin{array}{c}5 \\
\text { Gefühlte Sicherheit } \\
\text { (Instandhalter und System } \\
\text { hat alles im Blick) }\end{array}$}} & \multicolumn{2}{|c|}{16} & \multicolumn{2}{|c|}{15} \\
\hline & Am höchsten priorisierter Nutzenaspekt & & & Leistungsv & chnung & $\begin{array}{l}\text { Erhöhung de } \\
\text { und Anlagen }\end{array}$ & $\begin{array}{l}\text { aschinen- } \\
\text { ügbarkeit }\end{array}$ \\
\hline & Anzahl relevanter Risikogruppen & & & 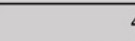 & & & \\
\hline & Am höchsten priorisierte Risikogruppe(n) & Risiko für da & nternehmen & $\begin{array}{r}\text { Dater } \\
\text { Voraussa }\end{array}$ & $\begin{array}{l}\text { ad } \\
\text { ualität }\end{array}$ & Kosten \& F & r Mensch \\
\hline Risiko & Anzahl relevanter Risikoaspekte & & & 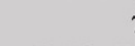 & & & \\
\hline KUSIKO & Am höchsten priorisierter Risikoaspekt & $\begin{array}{l}\text { Zuverläs } \\
\text { Genaui } \\
\text { Vorh }\end{array}$ & $\begin{array}{l}\text { reit und } \\
\text { it der } \\
\text { gen }\end{array}$ & $\begin{array}{l}\text { Erhöhte Fe } \\
\text { Störanfäll } \\
\text { Maschinen } \\
\text { zusätzlich }\end{array}$ & $\begin{array}{l}\text { r- bzw. } \\
\text { eit von } \\
\text { h bspw. } \\
\text { nsoren }\end{array}$ & $\begin{array}{r}\text { Einsparung } \mathrm{b} \\
\mathrm{PdM} \text { tritt nicl } \\
\mathrm{Höh}\end{array}$ & $\begin{array}{l}\text { MG durch } \\
\text { erwartetet } \\
\text { n }\end{array}$ \\
\hline
\end{tabular}

Abb. 5 Fallübergreifende Analyse von PdM (Predictive Maintenance). APU Auxiliary Power Unit, FMG Flughafen München GmbH, $P C A$ Pre Conditioned Air

\section{Schlussbetrachtung}

Von den drei untersuchten Anlagen am Flughafen München - Multienteiser, PCA-Anlagen und Aufzüge - weisen Aufzüge das höchste wirtschaftliche und qualitative Potenzial für die Einführung von PdM auf. Während PdM auch bei den PCA-Anlagen insbesondere aus qualitativer Sicht sinnvoll wäre, ist es für die Multienteiser gegenüber der traditionellen, erfahrungsbasiert optimierten Instandhaltung nicht von Vorteil.

Übergreifend zeigen die Ergebnisse der Studie, dass die Sinnhaftigkeit der Einführung einer PdM-Strategie durch eine Vielzahl von Faktoren beeinflusst wird, deren Ausprägung zwischen Anlagenklassen enorm variieren. Die Vorteilhaftigkeit sollte daher im Vorfeld der Einführung, vorzugsweise mithilfe des Verfahrens von Tauterat [6], geprüft werden. Während die Instandhaltungskosten in der Regel reduziert und die Anlagenverfügbarkeit erhöht werden kann, entstehen neuartige Risiken im Zusammenhang mit der Datensicherheit und eine erhöhte Störanfälligkeit aus zusätzlicher Sensorik. Im Falle einer Fremdinstandhaltung ist es zudem notwendig, die PdM-Strategie im Dienstvertrag zu verankern. Dies kann im Rahmen einer Ausschreibung zu einer eingeschränkten Anbieterauswahl führen und birgt außerdem ein Akzeptanzrisiko, da sich die Mitarbeiter durch das PdM-System kontrolliert fühlen könnten. Um für eigene Anlagen eine erste Indikation für die Eignung von PdM durchzuführen, können die in Tab. 7 abgebildeten Nutzen- und Risikofaktoren herangezogen werden. Hier handelt es sich um diejenigen Faktoren, die in der Fallstudie bei mindestens zwei Anlagen als relevant eingestuft wurden. Somit ist es eine priorisierte Sammlung der Aspekte aus [6]:

Vorliegender Beitrag ist eine der ersten Forschungsarbeiten, welche die Sinnhaftigkeit der Einführung einer PdMStrategie für unterschiedliche Anlagen anhand qualitativer und quantitativer Aspekte betrachtet. Entscheidungsträger in Unternehmen erhalten eine Orientierungshilfe, für welche Anlagenklassen PdM profitabel ist und welche Aspek-

Tab. 7 Nutzen- und Risikofaktoren zur Abschätzung der Eignung einer Anlagenklasse für PdM (Predictive Maintenance)

\begin{tabular}{|c|c|}
\hline Nutzenfaktoren & Risikofaktoren \\
\hline $\begin{array}{l}\text { Anlagen-, Maschinen- und } \\
\text { Bauteilverfügbarkeit }\end{array}$ & Daten- und Voraussagequalität \\
\hline Kosteneinsparung (In- & Datensicherheit (Datenmar \\
\hline
\end{tabular}

standhaltungs- und Energiekosten)

Personaleinsatz (Eigene und fremde Instandhaltungsmitarbeiter)

Planungssicherheit (Stillstandzeiten, optimierte Tourenplanung) Prozess- und Produktionsqualität erhöhte Anzahl an Schnittstellen)

Faktor Mensch (Mitarbeiterakzeptanz und Fehlinterpretation der Daten)

Kosten (PdM-System, Verlust von Garantieansprüchen)

Risiken des PdM-Systems (Erhöhte Fehler- bzw. Störanfälligkeit durch zusätzliche Sensoren) 
te in die ökonomische Bewertung grundsätzlich einbezogen werden müssen. Das angewandte Bewertungsverfahren dient als Muster für die Analyse weiterer technischer Anlagen und lässt sich auch für andere Technologien, wie beispielsweise Augmented Reality oder Cloud Computing, adaptieren. Zunächst müssten hierzu allerdings spezifische Aufwands-, Nutzen- und Risikoaspekte ermittelt werden.

Die Generalisierbarkeit der Ergebnisse ist dadurch eingeschränkt, dass drei Anlagen im selben Umfeld untersucht wurden. Außerdem beruhen die einzelnen Bewertungen vorwiegend auf Schätzungen der Workshopteilnehmer, was deren Validität begrenzt. Um generalisierbarere Schlüsse für die Eignung einer bestimmten Anlagenklasse für PdM ziehen zu können, ist es daher notwendig, die vorliegenden Ergebnisse in weiteren Forschungsarbeiten um die Analyse zusätzlicher Anlagen aus anderen Unternehmen und Branchen zu erweitern sowie zusätzliche Personengruppen (Softwareentwickler, IT-SicherheitVerantwortliche und das zuständige Betriebspersonal) in die Bewertung einzubeziehen.

Als Gesamtfazit lässt sich festhalten: PdM bietet Unternehmen ein erhebliches Potenzial. Allerdings ist es in Anbetracht der durchaus hohen initialen Investitionskosten nicht für jede Anlagenklasse gegenüber der vorausbestimmten und korrektiven Instandhaltung im Vorteil. Deshalb ist es unabdingbar, die Vorteilhaftigkeit im Einzelfall vor der Einführung genauestens zu prüfen.

Funding Open Access funding enabled and organized by Projekt DEAL.

Open Access Dieser Artikel wird unter der Creative Commons Namensnennung 4.0 International Lizenz veröffentlicht, welche die Nutzung, Vervielfältigung, Bearbeitung, Verbreitung und Wiedergabe in jeglichem Medium und Format erlaubt, sofern Sie den/die ursprünglichen Autor(en) und die Quelle ordnungsgemäß nennen, einen Link zur Creative Commons Lizenz beifügen und angeben, ob Änderungen vorgenommen wurden.

Die in diesem Artikel enthaltenen Bilder und sonstiges Drittmaterial unterliegen ebenfalls der genannten Creative Commons Lizenz, sofern sich aus der Abbildungslegende nichts anderes ergibt. Sofern das betreffende Material nicht unter der genannten Creative Commons Lizenz steht und die betreffende Handlung nicht nach gesetzlichen Vorschriften erlaubt ist, ist für die oben aufgeführten Weiterverwendungen des Materials die Einwilligung des jeweiligen Rechteinhabers einzuholen.

Weitere Details zur Lizenz entnehmen Sie bitte der Lizenzinformation auf http://creativecommons.org/licenses/by/4.0/deed.de.

\section{Literatur}

1. Amari SV, McLaughlin L, Pham H (2006) Cost-effective conditionbased maintenance using Markov decision processes. In: Annual reliability and maintainability symposium Newport Beach

2. Lee J, Qiu H, Ni J, Djurdjanovic D (2004) Infotronics technologies and predictive tools for next-generation maintenance systems. In: 11th IFAC symposium on information control problems in manufacturing Salvador
3. Labib AW (2004) A decision analysis model for maintenance policy selection using a CMMS. J Qual Maintenance Eng 10(3):191-202

4. Shin J-H, Jun H-B (2015) On condition based maintenance policy. J Comput Des Eng 2(2):119-127

5. Prajapati A, Bechtel J, Ganesan S (2012) Condition based maintenance: a survey. J Qual Maintenance Eng 18(4):384-400

6. Tauterat T (2018) Verfahren zur Bewertung von Predictive Maintenance für Anbieter von Instandhaltungsdienstleistungen. EUL Verlag, Siegburg

7. DIN Deutsches Institut für Normung: Instandhaltung - Begriffe der Instandhaltung; Dreisprachige Fassung EN 13306:2017 (2017)

8. Chebel-Morello B, Nicod J-M, Varnier C (2017) From prognostics and health systems management to predictive maintenance 2: knowledge, reliability and decision. John Wiley \& Sons, Hoboken

9. Jardine AK, Lin D, Banjevic D (2006) A review on machinery diagnostics and prognostics implementing condition-based maintenance. Mech Syst Signal Process 20(7):1483-1510

10. Alaswad S, Xiang Y (2017) A review on condition-based maintenance optimization models for Stochastically deteriorating system. Reliab Eng Syst Saf 157:54-63

11. Tang D, Makis V, Jafari L, Yu J (2015) Optimal maintenance policy and residual life estimation for a slowly degrading system subject to condition monitoring. Reliab Eng Syst Saf 134:198-207

12. Ye ZS, Xie M (2015) Stochastic Modelling and Analysis of Degradation for Highly Reliable Products. Appl Stoch Model Bus Ind 31(1):16-32

13. Si X-S, Wang W, Hu C-H, Zhou D-H (2011) Remaining useful life estimation-a review on the statistical data driven approaches. Eur J Oper Res 213(1):1-14

14. Neves ML, Santiago LP, Maia CA (2011) A condition-based maintenance policy and input parameters estimation for deteriorating systems under periodic inspection. Comput Ind Eng 61(3):503-511

15. Jeong I, Leon V, Villalobos J (2007) Integrated decision-support system for diagnosis, maintenance planning, and scheduling of manufacturing systems. Int J Prod Res 45(2):267-285

16. Ahmad R, Kamaruddin S (2012) A review of condition-based maintenance decision-making. Eur J Ind Eng 6(5):519-541

17. Sharma S, Cui Y, He Q, Mohammadi R, Li Z (2018) Data-driven optimization of railway maintenance for track geometry. Transp Res Part C Emerg Technol 90:34-58

18. Tang D, Yu J, Chen X, Makis V (2015) An optimal condition-based maintenance policy for a degrading system subject to the competing risks of soft and hard failure. Comput Ind Eng 83:100-110

19. Li X, Cai J, Zuo H, Li H (2017) Optimal cost-effective maintenance policy for a helicopter gearbox early fault detection under varying load. Mathematical Problems in Engineering. https://doi. org/10.1155/2017/4682409

20. Yildirim M, Sun XA, Gebraeel NZ (2016) Sensor-driven condition-based generator maintenance scheduling — part I: maintenance problem. IEEE Trans Power Syst 31(6):4253-4262

21. Pape U (2018) Investitionsrechnung. https://wirtschaftslexikon. gabler.de/definition/investitionsrechnung-41465/version-264829. Zugegriffen: 24. Juni 2019

22. Webster J, Watson RT (2002) Analyzing the past to prepare for the future: writing a literature review. Manag Inf Syst Q 26(2):13-26

23. Nemeth T, Ansari F, Sihn W, Haslhofer B, Schindler A (2018) Prima-X: a reference model for realizing prescriptive maintenance and assessing its maturity enhanced by machine learning. Proc CIRP 72:1039-1044

24. De Carolis A, Macchi M, Negri E, Terzi S (2017) A maturity model for assessing the digital readiness of manufacturing companies. In: IFIP international conference on advances in production management systems Hamburg

25. De Carolis A, Macchi M, Negri E, Terzi S (2017) Guiding manufacturing companies towards digitalization. A methodology for supporting manufacturing companies in defining their digitalizati- 
on roadmap. In: International conference on engineering, technology and innovation Madeira

26. Tafvizi Zavareh M, Sadaune S, Siedler C, Aurich JC, Zink KJ, Eigner M (2018) A Study on the Socio-Technical Aspects of Digitization Technologies for Future Integrated Engineering Work Systems. In: Nord Design 2018

27. Yin RK (2017) Case study research and applications: design and methods. SAGE, Thousand Oaks
28. Benbasat I, Goldstein DK, Mead M (1987) The case research strategy in studies of information systems. Manag Inf Syst Q 11(3):369-386

29. Paré G (2004) Investigating information systems with positivist case research. CAIS 13(1):233-264 